\title{
Hierarchical Spectrum Sharing in Cognitive Radio Networks
}

\author{
Yang Yu \\ Wuhan No.2 Ship Development Design Institute, Wuhan, China
}

544738692@qq.com

Keywords: Dynamic spectrum sharing, cognitive radio, spectrum leasing, opportunistic spectrum access, microeconomic theory, equilibrium.

\begin{abstract}
In this paper, we consider the problem of hierarchical bandwidth sharing in cognitive radio (CR) environment. A primary service provider (PSP) can sell its available spectrum bandwidth to a secondary service provider (SSP). In contrast to the conventional schemes, the SUs served by the SSP can also share the unsold bands of the PSP through the approach of opportunistic spectrum access (OSA), which will introduce interference to the PUs and make the PSP suffer a profit loss due to the sensing imperfections in practical networks. Based on microeconomic theory, this problem of bandwidth allocation is formulated as a market model, and an equilibrium where both the PSP and SSP are satisfied with the amount of allocated bandwidth and the price is derived by using the concept of demand and supply functions.
\end{abstract}

\section{Introduction}

Future wireless communication networks envisage the challenge that the available spectrum is becoming increasingly scarce. Cognitive radio (CR) [1] technology can intelligently identify unused licensed bands and allow adaptive utilization of them as long as not causing unacceptable interference from cognitive or secondary users (SUs) to licensed or primary users (PUs).

In a CR environment, dynamic spectrum sharing is a challenging problem due to the requirements of not only "peaceful" co-existence between PUs and SUs but also high spectrum utilization. In order to achieve these two goals at the same time, various spectrum sharing schemes have been raised [2]-[3]. A SU might attempt to coexist with PUs through opportunistic spectrum access (OSA), as long as its presence goes unnoticed or does not adversely affect the PUs [2]. Alternatively, a SU might access licensed bands through cooperation with PUs. For example, a primary licensee may grant its available bands to a secondary service provider (SSP) for a fee [3].

By now, in spectrum leasing networks, SUs can only access the bands which the primary service provider (PSP) sells to the SSP. However, it is not efficient enough for improving spectrum utilization, especially in the scenarios where some of the spectrum bands reserved by the PSP are grey spaces or white spaces [4]. According to our observation, SUs might also exploit these spectrum opportunities through OSA. Unfortunately, OSA to these bands will introduce interference to the PUs and degrade their performance on these bands, which means the PSP will suffer a profit loss. Since both the PSP and SSP aim at maximizing their profit, the price and supply of bandwidths in the secondary market will change.

In this paper, we consider this hierarchical spectrum sharing problem in the system where a PSP sells part of its bands to a SSP and allows SUs share its remaining unsold bands through OSA. This problem is formulated as a market model proposed in the microeconomics [5]. The bandwidth demand and supply functions based on the utilities achieved by the PUs and SUs are derived. With these two functions, an equilibrium, which satisfies both the service providers, is provided as the closed form solution of the proposed hierarchical spectrum sharing scheme.

\section{System Description}

In this paper, we consider a CR network with a PSP, which shares its total spectrum of size $W$ with a SSP, as shown in Figure 1. For the sake of simplicity, we take the case when there only 
exists a single PU and a single SU for example. In the secondary market, the PSP sells part of its bandwidth $B_{S}$, which can be divided into $N_{S}$ orthogonal narrowband channels, each with the bandwidth $B_{S, i}\left(i=1, \ldots, N_{S}, \sum_{s=1}^{N_{s}} B_{s, i}=B_{S}\right)$, to the SSP. In this case, the PSP charges the SSP at the rate of $P$, which is the price in the secondary market. For price setting, the PSP will negotiate the price, $P$, and the amount of bandwidth to be sold, $B_{S}$, with the SSP. And this negotiation repeats until the PSP and SSP are satisfied with the solution. Through the approach of OSA, the SU can also exploits the remaining unsold spectrum bandwidth of size $B_{P}=W-B_{S}$, which can be divided into $N_{P}$ orthogonal narrowband channels, each with the bandwidth $B_{P, j}\left(j=1, \ldots, N_{P}, \sum_{p=1}^{N_{p}} B_{P, j}=B_{P}\right)$.

We first consider the SU exploits communication opportunities within the remaining unsold bandwidth of the PSP. The SU decides whether or not to transmit on each channel based on the spectrum sensing results. If the $j$ th $\left(j=1, \ldots, N_{P}\right)$ channel is detected to be idle, the SU transmits using power $P_{S, j}$, whereas if the $j$ th channel is sensed to be active, then the SU does not transmit, in order not to introduce interference to the PU. However, due to the fact that spectrum sensing is not perfect in practical CR networks, the PU could either be miss-detected or a false alarm may occur. Correspondingly, four different cases are distinguished with respect to the sensing decision (present or absent) and the actual status of the PU (active or idle) on each channel. Therefore, there are four different instantaneous transmission rates of the SU on the $j$ th channel given as follows

$$
\begin{aligned}
& r_{S, j, 00}=B_{P, j} \log _{2}\left(1+\frac{P_{S, j} g_{S S}}{\sigma^{2}}\right), \\
& r_{S, j, 10}=B_{P, j} \log _{2}\left(1+\frac{P_{S, j} g_{S S}}{\sigma^{2}+P_{P, j} g_{P S}}\right), \\
& r_{S, j, 01}=r_{S, j, 11}=0,
\end{aligned}
$$

where the first index number of the instantaneous transmission rates describes the actual status of the PU (" 0 " for idle and " 1 " for active) and the second index number describes the decision which is made by the SU (" 0 " for absent and " 1 ” for present); $P_{P, j}$ denotes the transmit power of the PU on the $j$ th channel, $g_{S S}, g_{P S}$ and $g_{S P}$ denotes the instantaneous channel coefficients of the secondary link, the link between PU transmitter and SU receiver, and the link between SU transmitter and PU receiver, as shown in Figure 2, and $\sigma^{2}$ is the noise power.

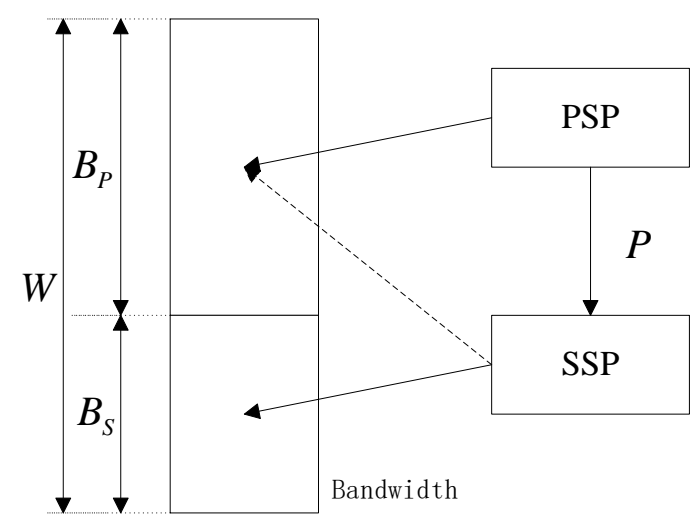

Fig. 1. The structure of hierarchical spectrum sharing system. 


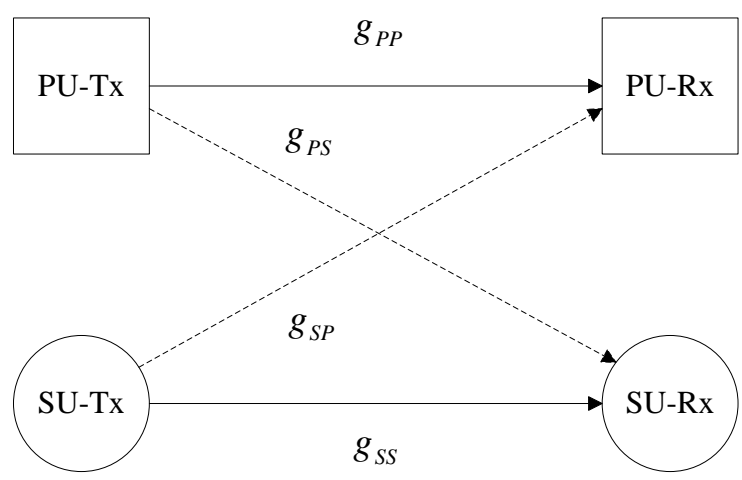

Fig. 2. System model for opportunistic spectrum sharing.

Similarly, the instantaneous transmission rates of the PU can be expressed as

$$
\begin{aligned}
& r_{P, j, 11}=B_{P, j} \log _{2}\left(1+\frac{P_{P, j} g_{P P}}{\sigma^{2}}\right), \\
& r_{P, j, 10}=B_{P, j} \log _{2}\left(1+\frac{P_{P, j} g_{P P}}{\sigma^{2}+P_{S, j} g_{S P}}\right), \\
& r_{P, j, 00}=r_{P, j, 01}=0,
\end{aligned}
$$

respectively, where $g_{P P}$ denotes the instantaneous channel coefficient of the primary link.

Thus, the average transmission rates of the $\mathrm{PU}$ and the SU on the $j$ th channel for the opportunistic spectrum sharing model can be written as

$$
\begin{aligned}
R_{S, j} & =\mathcal{P}\left(H_{0, j}\right)\left(1-\mathcal{P}_{f, j}\right) r_{S, j, 00}+\mathcal{P}\left(H_{0, j}\right) \mathcal{P}_{f, j} r_{s, j, 01} \\
& +\mathcal{P}\left(H_{1, j}\right)\left(1-\mathcal{P}_{d, j}\right) r_{S, j, 10}+\mathcal{P}\left(H_{1, j}\right) \mathcal{P}_{d, j} r_{s, j, 11}, \\
R_{P, j} & =\mathcal{P}\left(H_{0, j}\right)\left(1-\mathcal{P}_{f, j}\right) r_{P, j, 00}+\mathcal{P}\left(H_{0, j}\right) \mathcal{P}_{f, j} r_{P, j, 01} \\
& +\mathcal{P}\left(H_{1, j}\right)\left(1-\mathcal{P}_{d, j}\right) r_{P, j, 10}+\mathcal{P}\left(H_{1, j}\right) \mathcal{P}_{d, j} r_{P, j, 11},
\end{aligned}
$$

where $\mathcal{P}\left(H_{0, j}\right)$ and $\mathcal{P}\left(H_{1, j}\right)$ denotes the probability that the $j$ th channel is idle and active, $\mathcal{P}_{d, j}$ and $\mathcal{P}_{f, j}$ denotes the probability of detection and false alarm for the $j$ th channel.

For the transmission on the bandwidth which the PSP has sold to the SSP, since the primary signal is always absent, the SU can transmit all the time. Thus, the transmission rate of the SU using transmit power $\bar{P}_{S, i}$ on the $i$ th $\left(i=1, \ldots, N_{S}\right)$ channel is given by

$$
R_{S, i}=B_{S, i} \log _{2}\left(1+\frac{\bar{P}_{S, i} g_{S S}}{\sigma^{2}}\right),
$$

Therefore, the overall throughput of the PU and the SU is given by

$$
\begin{gathered}
R_{S}=\sum_{j=1}^{N_{P}} R_{S, j}+\sum_{i=1}^{N_{S}} R_{S, i}, \\
R_{P}=\sum_{j=1}^{N_{P}} R_{P, j} .
\end{gathered}
$$

\section{Algorithm Development}

This section formulates the problem of hierarchical spectrum sharing, and develops the proposed bandwidth allocation scheme. We consider a utility function to quantify user satisfaction as a function of transmission rate $R$ as follows [6]

$$
U(R)=\ln (R)+d,
$$


where $d$ is a constant. In this paper, we only consider the special case of $d=0$ to simplify our analysis. The following analytical procedure is suitable for any other values of $d$.

\subsection{Supply Function}

The supply function can be obtained based on profit maximization. The profit of the PSP is derived from the utility of the associated PU, and the revenue received from selling bandwidth to the SSP. The profit function is defined as follows

$$
\pi_{P}=U\left(R_{P}\right)+P B_{S}=\ln \left(R_{P}\right)+P B_{S} .
$$

Without loss of generality, we assume

$$
\mathcal{P}\left(H_{0, j}\right)=\mathcal{P}\left(H_{0}\right), \mathcal{P}\left(H_{1, j}\right)=\mathcal{P}\left(H_{1}\right), \mathcal{P}_{d, j}=\mathcal{P}_{d} \text { and } \mathcal{P}_{f, j}=\mathcal{P}_{f}, \text { for } j=1, \ldots, N_{P},
$$

to facilitate our discussion. Further, we also set the transmit power of the PU on different channel is the same, and so is the transmit power of the SU, i.e. $P_{P, j}=P_{P}, P_{S, j}=P_{S}$ and $\bar{P}_{S, i}=\bar{P}_{S}$ for $j=1, \ldots, N_{P}$ and $i=1, \ldots, N_{S}$.

Substituting (4)-(6), (8) into (11), and defining the constant

$$
\begin{aligned}
C_{1} & =\mathcal{P}\left(H_{1}\right)\left(1-\mathcal{P}_{d}\right) \log _{2}\left(1+\frac{P_{P} g_{P P}}{\sigma^{2}+P_{S} g_{S P}}\right) \\
& +\mathcal{P}\left(H_{1}\right) \mathcal{P}_{d} \log _{2}\left(1+\frac{P_{P} g_{P P}}{\sigma^{2}}\right),
\end{aligned}
$$

We can obtain

$$
R_{P}=\sum_{j=1}^{N_{P}} C_{1} B_{P, j}=C_{1} B_{P} .
$$

Therefore, we can rewrite profit function (13) as

$$
\pi_{P}=\ln \left(C_{1} B_{P}\right)+P B_{S} .
$$

The supply function can be obtained based on maximizing profit of the PSP, i.e.

$$
S=B_{S}^{*}=\arg \max _{B_{S}} \pi_{P} .
$$

By differentiating profit function (15) with respect to $B_{S}$, we can obtain

$$
\frac{\partial \pi_{P}}{\partial B_{S}}=\frac{1}{B_{S}-W}+P=0
$$

Solving the equation (17), the supply function for the market price $P$ can be expressed as follows

\subsection{Demand Function}

$$
S=W-\frac{1}{P} \text {. }
$$

To derive the demand function, we again utilize the concept of profit maximization. This profit function of SSP is defined as follows

$$
\pi_{S}=U\left(R_{S}\right)-P B_{S} .
$$

According to (1)-(3), (7), (9), (10), and defining the following constants

$$
\begin{aligned}
C_{2} & =\mathcal{P}\left(H_{0}\right)\left(1-\mathcal{P}_{f}\right) \log _{2}\left(1+\frac{P_{S} g_{S S}}{\sigma^{2}}\right) \\
& +\mathcal{P}\left(H_{1}\right)\left(1-\mathcal{P}_{d}\right) \log _{2}\left(1+\frac{P_{S} g_{S S}}{\sigma^{2}+P_{P} g_{P S}}\right) \text { and } C_{3}=\log _{2}\left(1+\frac{\bar{P}_{S} g_{S S}}{\sigma^{2}}\right),
\end{aligned}
$$

We can obtain

Then we can rewrite (19) as

$$
R_{S}=\sum_{j=1}^{N_{P}} C_{2} B_{P, j}+\sum_{i=1}^{N_{S}} C_{3} B_{S, i}=C_{2} B_{P}+C_{3} B_{S} .
$$




$$
\pi_{S}=\ln \left(C_{2} B_{P}+C_{3} B_{S}\right)-P B_{S} .
$$

Similarly, the demand function can be obtained as follows

$$
D=B_{S}^{*}=\arg \max _{B_{S}} \pi_{S}=\frac{1}{P}+\frac{C_{2} W}{C_{2}-C_{3}} .
$$

Since $S>0$ and $D>0$, the feasible region of $P$ is $\left(1 / W,\left(C_{3}-C_{2}\right) / C_{2} W\right)$.

\subsection{Equilibrium}

Given the supply and the demand functions, the equilibrium can be obtained. The equilibrium defines the quantity of product and the price such that demand is equal to supply, i.e., the SSP buys all of the supplied bandwidth. Therefore, we can obtain this equilibrium from $S=D$ as follows

$$
W-\frac{1}{P}=\frac{1}{P}+\frac{C_{2} W}{C_{2}-C_{3}} .
$$

At the equilibrium, the market price can be simply obtained by solving (23)

$$
P^{*}=\frac{2\left(C_{3}-C_{2}\right)}{W C_{3}} .
$$

After we get the market price at the equilibrium point, the amount of bandwidth to be sold to the SSP can be computed by (22).

It is obvious that the equilibrium can be obtained if all the information on price and bandwidth demand is available. However, this is not possible for a distributed network. In an actual market, this equilibrium is often obtained through the negotiation between the supplier and the consumer iteratively. Thus, we propose an iterative algorithm to achieve it.

In this algorithm, the supplier adjusts the price based on the excess demand information fed back from the consumer. In our hierarchical spectrum sharing system, the PSP randomly chooses initial price $P[0]$ and submits it to the SSP. The SSP replies to the PSP with the bandwidth demand. The PSP computes the excess demand by subtracting the bandwidth supply from the bandwidth demand received from the SSP. In particular, if the demand is larger than the supply, the supplier has to increase price to obtain higher revenue. This excess demand is weighted by the parameter $\alpha$ (learning rate) and added to the previous price. The algorithm is repeated until the stopping criterion is met, i.e., $|P[t+1]-P[t]|<\varepsilon$. An iterative expression for the price at time $t$ can be defined as follows:

$$
P[t+1]=P[t]+\alpha(D[t]-S[t]),
$$

where $S[t]=W-1 / P[t]$ and $D[t]=1 / P[t]+C_{2} W /\left(C_{2}-C_{3}\right)$.

\section{Simulation Results}

In this section, we present numerical results to illustrate the performance of our scheme. In the simulation, the total frequency spectrum available to the PSP is $10 \mathrm{MHz}$. The initial price setting for the secondary market is $P[0]=0.125$. The parameter used in the stopping criterion for the iterative algorithm is $\varepsilon=10^{-4}$. All the channel coefficients are set equal to 1 . The target detection and false alarm probability for all channels reserved by the PSP is set to be $\mathcal{P}_{d}=90 \%$ and $\mathcal{P}_{f}=5 \%$, respectively. We further assume $\mathcal{P}\left(H_{0}\right)=15 \%$. Finally, the transmit power of the PU on all its reserved channels is assumed to be $P_{P}=10 \mathrm{~dB}$, and the noise variance $\sigma^{2}$ is set to be 1 .

In Fig. 3, we first show bandwidth demand and supply functions in the secondary market with respect to the price for different transmit power $P_{S} . \bar{P}_{S}$ is set to be $10 \mathrm{~dB}$. As expected, the bandwidth supply increases as the price increases, since the PSP can achieve higher profit. The equilibriums of this bandwidth sharing are located at the point where the bandwidth supply and the demand functions intersect. These equilibriums can be obtained easily if all the information on demand and 
supply functions is available.

The dynamic behaviors (i.e., adaptation over time) of the iterative algorithm are shown in Fig. 4. Depending on the learning rate $\alpha$, the demand adaptation can be stable $(\alpha=0.003,0.007)$ or unstable ( $\alpha=0.033$ ). When the learning rate is properly set, the bandwidth demand converges to the equilibrium quickly. In the contrary, when the learning rate is large, the bandwidth demand fluctuates between two values. And the adaptation swings and never reaches the equilibrium.

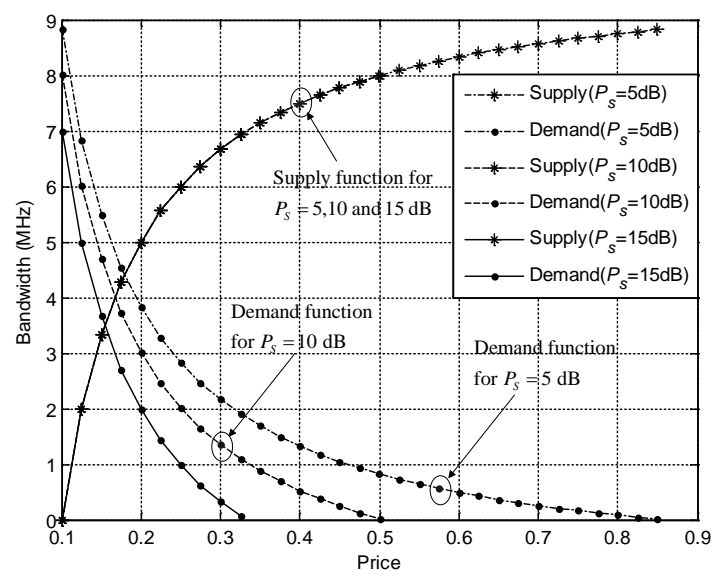

Fig. 3. Bandwidth demand and supply functions for different $P_{s}$.

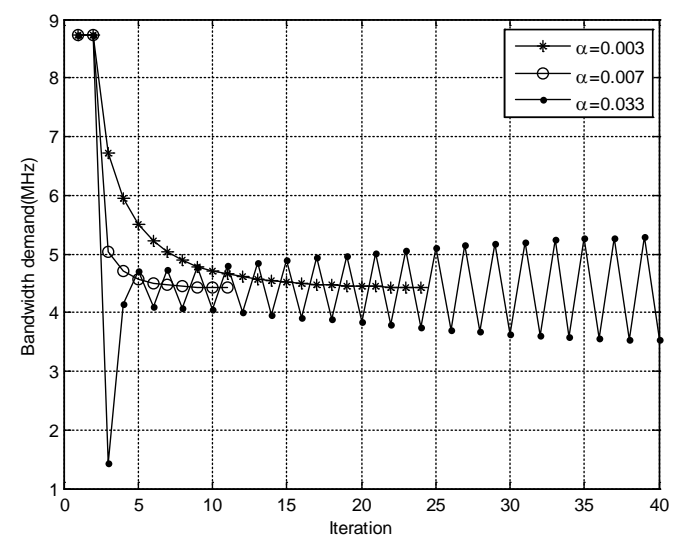

Fig. 4. Demand adaptation under different learning rate.

\section{Conclusion}

In this paper, we have considered the dynamic spectrum sharing problem between a PSP and a SSP in a CR network, where the SU can share the unsold bands of the PSP through the means of OSA. We have formulated this problem as a market model, and the bandwidth demand and supply functions have been derived from the utility of the PU and SU. The equilibrium is considered as the solution of this hierarchical spectrum sharing where the supply bandwidth is equal to the demand bandwidth. Achieving this equilibrium means there is no extra spectrum demand from the SSP and the PSP is satisfied with the price setting. An iterative algorithm to achieve the equilibrium has also been proposed when the global information is not available.

\section{References}

[1] A. Goldsmith, S. Jafar, I. Maric, and S. Srinivasa, "Breaking spectrum gridlock with cognitive radios: an information theoretic perspective,” Proc. IEEE, vol. 97, pp. 894-914, May. 2009. 
[2] J. C. F. Li, W. Zhang, and J. Yuan, "Opportunistic spectrum sharing in cognitive radio networks based on primary limited feedback,” IEEE Trans. Commun., vol. 59, no. 12, pp. 3272-3277, Dec. 2011.

[3] D. Niyato, P. Wang, and Z. Han, "Dynamic spectrum leasing and service selection in spectrum secondary market of cognitive radio networks," IEEE Trans. Wireless Commun., vol. 11, no. 3, pp. 1136-1145, Mar. 2012.

[4] S. Haykin, “Cognitive radio: brain-empowered wireless communication-s,” IEEE J. Sel. Areas Commun., vol. 23, no. 2, pp. 201-220, Feb. 2005.

[5] F. V. Waugh, “Cobweb Model,” J. Farm Economics, vol. 46, no. 4, pp. 732-750, 1964.

[6] A. Tang, J. Wang, and S. H. Low, “Counter-intuitive throughput behaviors in networks under end-to-end control,” IEEE/ACM Trans. Networking, vol. 14, no. 2, pp. 355-368, Apr. 2006. 